\title{
A multi-wavelength analysis of $M$ 81: insight on the nature of Arp's loop
}

\author{
A. Sollima ${ }^{1}$, A. Gil de Paz $^{2}$, D. Martinez-Delgado ${ }^{1,3}$, R. J. Gabany ${ }^{4}$, J. J. Gallego-Laborda ${ }^{5}$, and T. Hallas ${ }^{6}$ \\ 1 Instituto de Astrofisica de Canarias, La Laguna, E38205, Spain \\ e-mail: asollima@iac.es (AS) \\ ${ }^{2}$ Departamento de Astrofisica, Universidad Complutense de Madrid, E28040, Spain \\ 3 Max-Planck-Institut fur Astronomie, Heidelberg, Germany \\ 4 Blackbird Observatory, New Mexico, USA \\ Fosca Nit Observatory, Montsec Astronomical Park, Ager, Spain \\ ${ }^{6}$ Hallas Observatory Annex, Foresthill, USA
}

Received 18 January 2010 / Accepted 6 April 2010

\section{ABSTRACT}

\begin{abstract}
Context. The optical ring-like structure detected by Arp (1965) around M 81 (commonly referenced as "Arp's loop") represents one of the most spectacular features observed in nearby galaxies. Arp's loop is commonly interpreted as a tail resulting from the tidal interaction between M 81 and M 82. However, since its discovery the nature of this feature has remained controversial. Aims. Our primary purpose was to identify the sources of optical and infrared emission observed in Arp's loop.

Methods. The morphology of Arp's loop has been investigated with deep wide-field optical images. We also measured its colors using IRAS and Spitzer-MIPS infrared images and compared them with those of the disk of M 81 and Galactic dust cirrus that fills the area where M 81 is located.

Results. Optical images reveal that this peculiar object has a filamentary structure characterized by many dust features overlapping M 81's field. The ratios of far-infrared fluxes and the estimated dust-to-gas ratios indicate the infrared emission of Arp's loop is dominated by the contribution of cold dust that is most likely from Galactic cirrus.

Conclusions. The above results suggest that the light observed at optical wavelengths is a combination of emission from i) a few recent star-forming regions located close to M 81, where both bright UV complexes and peaks in the HI distribution are found, ii) the extended disk of M 81 and iii) scattered light from the same Galactic cirrus that is responsible for the bulk of the far-infrared emission.
\end{abstract}

Key words. methods: data analysis - techniques: photometric - galaxies: individual: M 81 - infrared: galaxies

\section{Introduction}

The nearby galaxy M 81 (NGC 3031) together with the galaxies M 82, NGC 3077 and NGC 2976 forms one of the best local examples of a group of interacting galaxies. Located at a distance of $\sim 3.7 \mathrm{Mpc}$ (Makarova et al. 2002), the M 81 group has been a subject for many studies analyzing the evidence of strong interactions among its components. The group contains remnants of tidal bridges connecting the three most prominent galaxies, visible in neutral hydrogen (HI) survey (Gottesman \& Weliachew 1975; Yun et al. 1994), and over 40 dwarf galaxies (Karachentsev et al. 2001).

In particular, Arp (1965) detected an unusual ring-shaped feature in the vicinity of M 81 while examining Schmidt photographic plates. This optical feature (usually referenced as "Arp's loop") is located 17 arcmin north-east of M 81's center and covers a wide area of $\sim 160$ square arcmin. It also exhibits symmetry towards the end of the M 81 disk, slightly tilted toward M 82.

The Arp's loop is commonly interpreted as a tail resulting from the tidal interaction between M 81 and M 82. However, since its discovery this interpretation has been doubted by many authors. In fact, the region containing the M 81 group of galaxies is filled by Galactic cirrus that covers a large area of the sky near M 81 (Sandage 1976). Arp's loop exhibits colors and emission properties similar to those observed in Galactic cirrus clouds (Abolins \& Rice 1987; Appleton et al. 1993; Bremnes et al. 1998), and this has raised doubts about its association with M 81.

Subsequent analyses supporting the extra-Galactic nature of Arp's loop were based on $21 \mathrm{~cm} \mathrm{HI}$ observations in the M 81 group region (Yun et al. 1994). These authors detected a collimated emission in the direction of the north-eastern part of Arp's loop which blends smoothly into the structure and velocity of the HI disk of M 81. Numerical simulations of the system by Yun (1999) successfully reproduced the HI tidal debris assuming M 82 and NGC 3077 approached M 81 during recent epochs. More recently, Makarova et al. (2002) and De Mello et al. (2008) resolved the nebular region characterized by strong $\mathrm{HI}$ emission into stars using deep Hubble Space Telescope (HST) observations. Their color-magnitude diagrams (CMD) showed a significant population of young stars (with an age of 40-160 Myr) and an old population characterized by a well defined Red Giant Branch. The magnitude of the tip of the Red Giant stars assumed to be associated with Arp's loop indicated a distance comparable with that of M 81 (Karachentsev et al. 2002). These authors suggested that the old component could have been removed from the M 81 and/or M 82 disk during their mutual interaction. Further insight was recently provided by Barker et al. (2009), who analyzed a wide area that included M 81 with deep Suprime-Cam observations. Their results revealed no overdensity of Red Giant 
stars along Arp's loop extension (which is at odds with De Mello et al.'s results), whereas a significant group of young Red Supergiants and Main Sequence stars were evident in the confined region previously surveyed in HST studies. These authors concluded that Arp's loop was originally a gaseous tidal debris stream that formed stars only in the last 200-300 Myr. The same qualitative results have also been obtained by Davidge (2009).

In this paper we present the results of a multi-wavelength analysis of Arp's loop using the deepest optical and infrared observations available.

\section{The data}

Our analysis is based on three datasets: i) a set of deep groundbased optical images obtained at different facilities (the Fosca Nit Observatory and Hallas Observatory), a set of far-infrared images observed with the Multiband Imaging Photometer for Spitzer (MIPS) at the Spitzer Space Telescope at $24 \mu \mathrm{m}, 70 \mu \mathrm{m}$ and $160 \mu \mathrm{m}$ and with IRAS using the latest IRIS data products and iii) a set of high-resolution HI maps constructed from VLA interferometric observations (from the The HI Nearby Galaxy Survey (THINGS) database; Walter et al. 2008).

The first dataset was collected with a commercially available $106 \mathrm{~mm}$ aperture $f / 5$ Takahashi FSQ refractor telescope of the Fosca Nit Observatory (FNO) situated near Ager (Spain) at the Montsec Astronomical Park. We used a Santa Barbara Imaging Group (SBIG) STL-11000M CCD camera which yields a large field-of-view $\left(3.9^{\circ} \times 2.7^{\circ}\right)$ at a plate scale of $3.5 \mathrm{arcsec} / \mathrm{pixel}$. The image set consists of multiple deep exposures through four Optec Inc. broadband (LBGR) filters. A set of individual $600 \mathrm{~s}$ images were obtained during several photometric nights between January and February 2008, achieving a total exposure time of the co-added images of $17100 \mathrm{~s}$.

High-resolution images were also gathered during four photometric nights between February and March 2007 with a $14.5^{\prime \prime} \mathrm{f} / 8$ RCOS cassegrain telescope situated at the Hallas Observatory Annex (HOA) situated near Foresthill, California (USA). A Santa Barbara Imaging Group STL-11000M camera, yielding a $41^{\prime} \times 27^{\prime}$ field-of-view and a 1 arcsec/pixel plate scale, was attached to the Cassegrain focus of the telescope. A set of images through four broadband (LGBR) filters were secured and co-added, yielding a total exposure time of $51400 \mathrm{~s}$.

The images were reduced following standard procedures for bias correction and flat-fielding. To enhance the signal-to-noise of the faint structures around M 81, image noise was attenuated with a Gaussian filter (Davis Davis).

Multiband imaging photometer for Spitzer (MIPS) images were also retrieved from the Spitzer Infrared Nearby Galaxy Survey (SINGS Data Release 4, Kennicut et al. 2003) public archive. The sample consists of a set of deep images in the $24 \mu \mathrm{m}, 70 \mu \mathrm{m}$ and $160 \mu \mathrm{m}$ bands. The total exposure time is approximately $220 \mathrm{~s}, 84 \mathrm{~s}$ and $25 \mathrm{~s}$ at $24 \mu \mathrm{m}, 70 \mu \mathrm{m}$ and $160 \mu \mathrm{m}$, respectively. Individual frames were reduced with the MIPS Instrument Team Data Analysis Tool (Gordon et al. 2005). The background was subtracted using the average value of an empty region of the MIPS field of view. The uncertainties of the final absolute calibrations were estimated at $10 \%$ for the $24 \mu \mathrm{m}$ data and $20 \%$ for both the $70 \mu \mathrm{m}$ and $160 \mu \mathrm{m}$ data. The combined images were then aligned in the standard WCS reference frame. The overall field-of-view is approximately $30^{\prime} \times 44^{\prime}$.

To compare the colors of Arp's loop with those of nearby Galactic cirrus we also retrieved the newest generation IRIS imaging products from the IRAS satellite covering a large region $\left(12.5^{\circ} \times 12.5^{\circ}\right)$ around $\mathrm{M} 81$ (see Fig. 3 ). The IRIS images that we

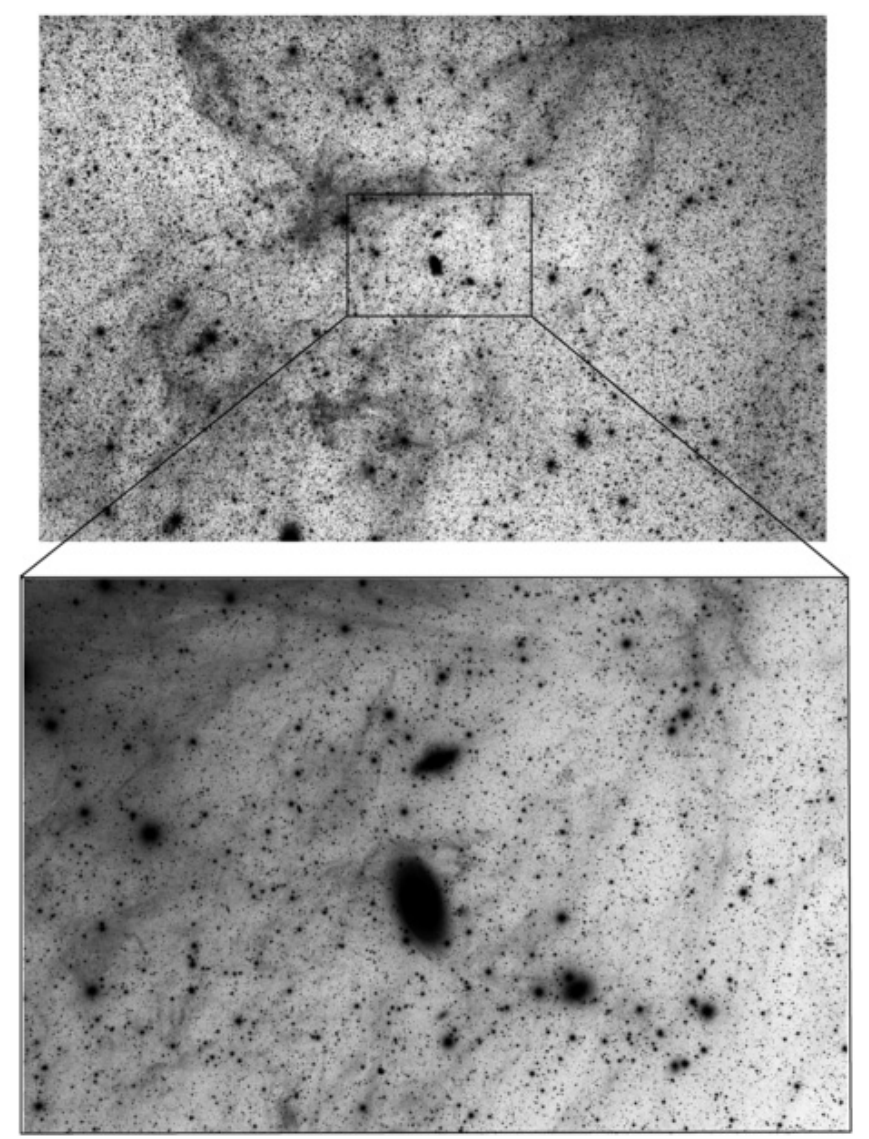

Fig. 1. Upper panel: $2^{\circ} \times 3^{\circ}$ image of the $\mathrm{M} 81$ region from Witt et al. (2008; by courtesy of S. Mandel). North is up, east to the right. Bottom panel: FNO image of the M 81 group of galaxies.

used benefit from better zodiacal-light subtraction, calibration, zero levels, and destriping than previous versions. In particular, the $100 \mu \mathrm{m}$ IRIS maps represent a significant improvement over those used by Schlegel et al. (1998).

Neutral hydrogen maps were retrieved from the THINGS archive. The data for M 81 consist in a set of high-resolution $21 \mathrm{~cm}$ observations performed with the VLA array of radio telescopes. Observations were performed in B, C, and D configuration with a bandwidth of $1.56 \mathrm{MHz}$. The calibration and data reduction were performed with standard routines in the AIPS package. A high-resolution map was constructed adopting a beam size of $12.91^{\prime \prime} \times 12.41^{\prime \prime}$. The overall spatial resolution of the final map is $6^{\prime \prime}$ with a pixel size of $1.5^{\prime \prime}$. A detailed description of the data reduction procedure can be found in Walter et al. (2008).

\section{Results}

\subsection{Optical imaging}

Figure 1 (bottom panel) shows a wide-field view around M 81 obtained with the FNO telescope. This image clearly shows many large-scale cirrus structures. The width of these filaments range from $\sim 30$ arcsec up to several arc minutes and extend in connective patterns over several degrees (as it is clearly visible in the panorama of this sky region displayed in the image obtained by Witt et al. 2008; see Fig. 1, top panel).

Figure 2 displays the image obtained by combining all the HOA observations. This image covers an area of $41^{\prime} \times 27^{\prime}$. This deep image reaches a point-source limiting visual magnitude of 


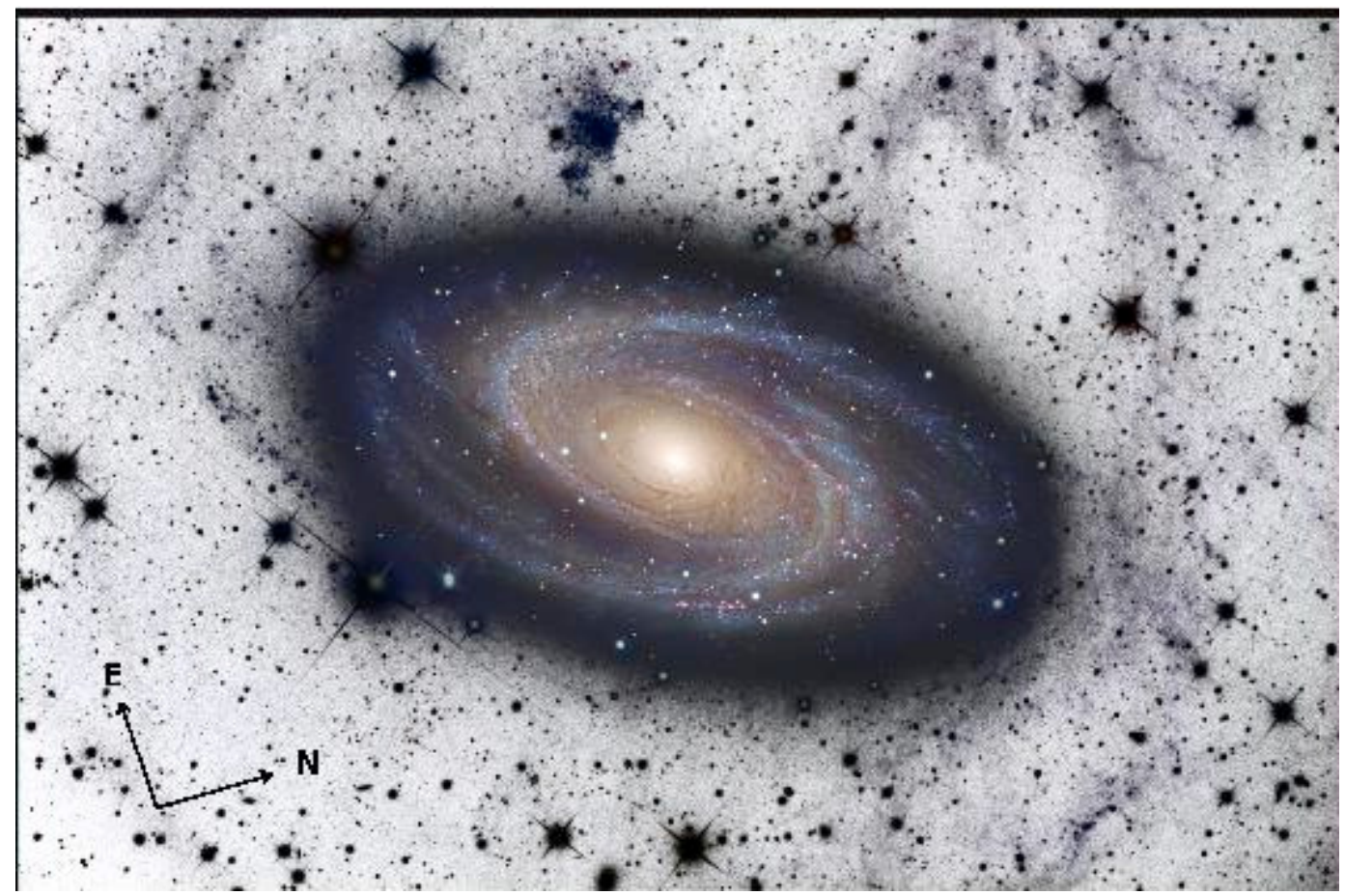

Fig. 2. Combined HOA image of the M 81 region. The field of view of this image is $41^{\prime} \times 27^{\prime}$. The north and east directions are indicated. Arp's loop is visible as the nebular ring crossing the disk of M 81 on the right side of the image. Dust-absorbtion features are superimposed over and surround the disk of M 81 .

$V \sim 27$. The spectacular structure of Arp's loop is evident in the north-eastern region. Arp's loop appears as a dim filamentary ring that wraps and overlaps the galaxy's disk. A careful inspection of M 81's disk reveals several dust absorption features that correspond with the intersection of Arp's loop and the galaxy. These features, already noticed by Arp (1965) and Efremov et al. (1986), are due to dust in the ring, indicating that part of it should be situated between the observer and M 81. In the north-eastern part of this feature (in the region where HI strong emission was found by Yun et al. 1994) a significant overdensity of stellar complexes is also apparent and suggests that new stars are still forming (Makarova et al. 2002; Mouhcine \& Ibata 2010).

\subsection{Far-infrared emission}

As previously mentioned in Sect. 1, far-infrared imaging is a fundamental tool to highlight emission from dusty objects. This is particularly important when analyzing Arp's loop because both dust absorption in the form of dust lanes projected against the disk of M 81 and dust emission from the loop itself are clearly seen (see Sect. 3.1). The right panel of Fig. 3 shows an IRAS map in a wide region $\left(12.5^{\circ} \times 12.5^{\circ}\right)$ around the M 81 group of galaxies constructed using images in the $12 \mu \mathrm{m}, 60 \mu \mathrm{m}$ and $100 \mu \mathrm{m}$ bands. As noted in Fig. 1, the surveyed area is filled with Galactic cirrus clouds.

To compare Arp's loop with the cirrus clouds in infrared light, we measured the fluxes for various regions of the $60 \mu \mathrm{m}$ and $100 \mu \mathrm{m}$ IRAS image (see Fig. 3). Removal of the background (mainly of cosmic origin, because zodiacal light was previously removed from the IRIS images) was performed using the minimum value of the surface brightness in the $12.5^{\circ} \times 12.5^{\circ}$ IRIS image for each band. Despite its admittedly large amount of uncertainty, this method has been already used in the past (Carey et al. 1997) and yields a value for the background at $100 \mu \mathrm{m}(0.67 \mathrm{MJy} / \mathrm{sr})$, which is very similar to the average
Cosmic Infrared Background of $0.78 \mathrm{MJy} / \mathrm{sr}$ as determined by Lagache et al. (2000). The adopted apertures and the resulting fluxes for a number of regions in M 81 and the Arp's loop are listed in Table 1.

In the left panel of Fig. 3 the ratio between the $60 \mu \mathrm{m}$ and $100 \mu \mathrm{m}$ fluxes are plotted for the main body of M 81 (open black point), cirrus clouds (grey points) and Arp's loop (black filled points) as a function of the $100 \mu \mathrm{m}$ surface brightness. It should be noted that cirrus clouds occupy a well defined sequence in this diagram, with high-density cirrus showing a lower ratio than the most diffuse cirrus in the field. This trend has been previously reported by several authors (e.g. Abergel et al. 1994). For comparison, the "reference value" commonly adopted for the $I_{60 \mu \mathrm{m}} / I_{100 \mu \mathrm{m}}$ ratio in high-latitude cirrus is $\sim 0.2$ (see e.g. Arendt et al. 1998). M 81 presents a high color $\left(I_{60 \mu \mathrm{m}} / I_{100 \mu \mathrm{m}} \sim 0.3\right)$ compared to Galactic cirrus of the same brightness, although it is not very different from diffuse cirrus colors or from that of the loop itself. In this sense it is worth noting that M 81 is a very quiescent object in terms of its dust emission properties (see e.g. Dale et al. 2007). In fact, it has the lowest $I_{60 \mu \mathrm{m}} / I_{100 \mu \mathrm{m}}$ ratio among those in the Helou (1986) sample of galaxies. More importantly, the colors found within the circular apertures placed on the loop (cyan circles in Fig. 3) are not very different from the color of the M 81 disk and follow the same trend as the cirrus. This latter fact suggests a Galactic origin for the bulk of the far-infrared emission associated with Arp's loop.

Despite these results, it is still conceivable that dust in the outer disk of M81 or in a potential tidal feature around M 81 could show the same colors (and follow the same surface brightness trend) as the cirrus, because they could contain cold dust with emission properties similar to Galactic cirrus. The reader is referred to the recent work by Bot et al. (2009) for an extensive discussion on the properties of Galactic cirrus.

A deeper insight into the structure of dust emission around M 81 necessarily requires deep high-resolution (arcmin-scale) 


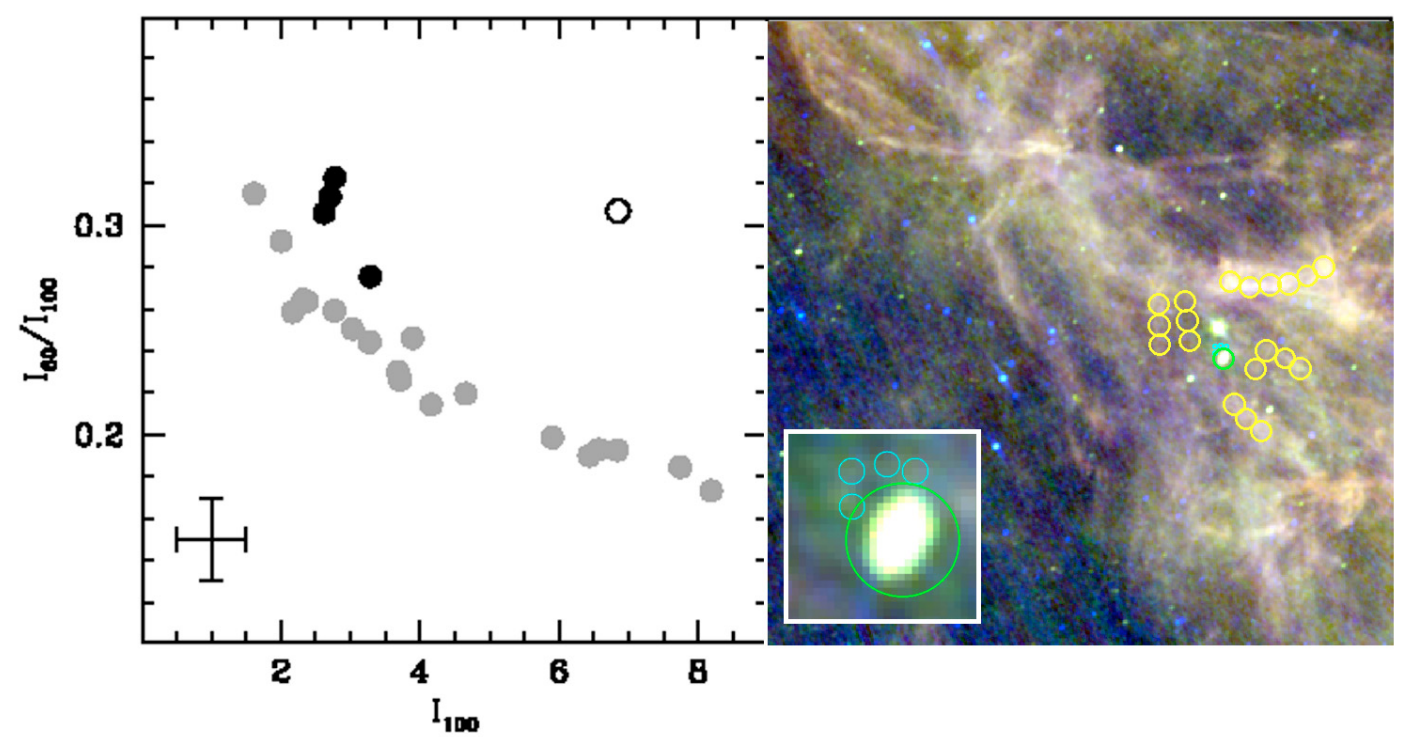

Fig. 3. In the left panel the $I_{60} \mu \mathrm{m} / I_{100 \mu \mathrm{m}}$ ratio is shown as a function of the $I_{100 \mu \mathrm{m}}$ brightness for the loop (black filled points), M 81 (open point) and the surrounding cirrus features (grey points). The typical uncertainty of the regions along Arp's loop is shown in the bottom part of the panel. The right panel depicts a false-color IRAS image of the M 81 region. North is up, east is to the left. The circular apertures used in the analysis of the Galactic cirrus (yellow circles; 12' diameter), M 81 (green circle; 12' diameter) and Arp's loop (tiny cyan circles above M 81; 4' diameter) are shown. A zoom into the M 81 region is shown in the inner box.

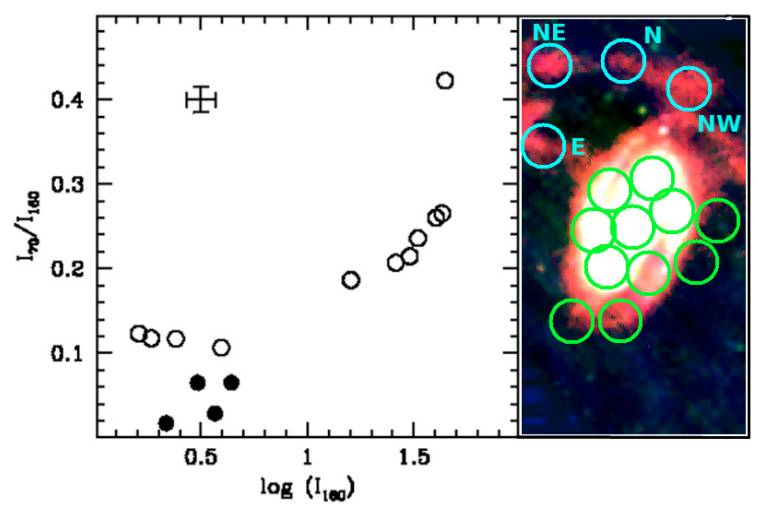

Fig. 4. In the left panel the $I_{70 \mu \mathrm{m}} / I_{160 \mu \mathrm{m}}$ ratio calculated for Arp's loop (filled circles) and the M 81 disk (open circles) are shown as a function of the $I_{160 \mu \mathrm{m}}$ brightness. The typical uncertainty is shown in the upper part of the panel. In the right panel the $27^{\prime} \times 41^{\prime}$ color map of the Spitzer MIPS emission for M 81 was constructed with the $24 \mu \mathrm{m}, 70 \mu \mathrm{m}$ and $160 \mu \mathrm{m}$ images. The circular apertures are also shown with the same color code as in Fig. 3.

Spitzer-MIPS observations. The MIPS $160 \mu \mathrm{m}$ data contain information at longer wavelengths, which might offer further clues about the possible differences (or similarities) between dust emission in the loop and M 81. The right panel of Fig. 4 displays a color-emission map from Spitzer. To construct this map, the MIPS/Spitzer $24 \mu \mathrm{m}$ and $70 \mu \mathrm{m}$ images were binned and scaled to the resolution of the $160 \mu \mathrm{m}$ image using convolution kernels developed by Gordon et al. (2008). To compare the farinfrared colors of Arp's loop with the stellar component of M 81 we measured the $70 \mu \mathrm{m}$ and $160 \mu \mathrm{m}$ fluxes sampled in circular apertures positioned at four regions of Arp's loop and across the M 81's disk (see the right panel of Fig. 4). We exercised care in selecting both regions populated by stellar complexes and regions where only an extended diffuse emission is noticeable from optical images along Arp's loop. The measured fluxes are listed in Table 1. Most importantly, the $70 \mu \mathrm{m} / 160 \mu \mathrm{m}$ flux ratio as a function of the $160 \mu \mathrm{m}$ brightness is shown in the left panel.
Note that Arp's loop and the disk of M 81 display very different colors. Here the segregation between M 81 and the loop is much clearer than it is in the IRAS $I_{60 \mu \mathrm{m}} / I_{100 \mu \mathrm{m}}$ ratio. In particular, the ratio $70 \mu \mathrm{m} / 160 \mu \mathrm{m}$ appears smaller $(<0.1$ in all cases) than that measured on the galaxy's disk (which typically ranges between $0.2-0.4$ in the inner disk and form a plateau around 0.12 in the outer disk) along the entire extension of the loop. The $70 \mu \mathrm{m} / 160 \mu \mathrm{m}$ ratios measured in the loop are also similar to the $60 \mu \mathrm{m} / 160 \mu \mathrm{m}$ ratios found by Bot et al. (2009) in their analysis of serendipitous observations of Galactic cirrus contained in the SINGS Spitzer-MIPS fields.

\subsection{Comparison between far-infrared and $\mathrm{HI}$ emission}

Figure 5 displays the map of the HI emission measured by Walter et al. (2008) and the MIPS $160 \mu \mathrm{m}$ one overplotted on the optical HOA image. Notice that the HI emission nicely follows the spiral arms of M 81. Also Holmberg IX (visible on the east of M 81 in Fig. 5) and the southern part of Arp's loop are embedded in clouds connected to the main body of M 81. On the other hand, most of the far-infrared emission appears to be associated to the disk of M 81, with a significant contribution from Arp's loop. In addition, note that the HI emission extends across the entire galaxy disk well beyond its optical extent, partially overlapping Arp's loop. It is worth noting that while the $160 \mu \mathrm{m}$ flux is relatively high along the entire loop extension, the HI emission disappears in the northern part. To better visualize this comparison, the contours of the HI emission measured by Walter et al. (2008) are overplotted to the MIPS $160 \mu \mathrm{m}$ image in Fig. 6. The mismatch between the spatial distribution of the HI and MIPS $160 \mu \mathrm{m}$ emission suggests that different regions of Arp's loop are characterized by different emitting mechanisms.

A more quantitative comparison was performed by estimating the relative fraction of dust and gas over Arp loop's extension and across the main body of M 81. This parameter is particularly powerful in distinguishing between the "canonical" emission of galactic disks and other dust-dominated sources. Indeed, this quantity spans a limited range among the observed galaxies 
and has been found to show a well defined radial behavior, decreasing at large distances from the galactic center (Issa et al. 1990; Boissier et al. 2004; Muñoz-Mateos et al. 2009). To estimate the total dust masses, we used MIPS fluxes at $24 \mu \mathrm{m}$, $70 \mu \mathrm{m}$ and $160 \mu \mathrm{m}$ measured in the same circular apertures along Arp's loop and across the M 81's disk defined in Sect. 3.2 (see also the right panel of Fig. 7). We adopted the relation by Muñoz-Mateos et al. (2009; see their Eq. (A8)) to convert fluxes in masses adopting a distance for M 81 of $3.7 \mathrm{Mpc}$ (Makarova et al. 2002). In order to obtain total gas masses, we measured the HI fluxes from the M 81 intensity map obtained by the THINGS survey (Walter et al. 2008). For this purpose the THINGS image has been binned and scaled to the resolution of the $160 \mu \mathrm{m}$ image using the convolution kernels developed by Gordon et al. (2008). The corresponding total gas masses were calculated using Eq. (3) of Walter et al. (2008). The resulting dust and HI masses together with the corresponding dust-to-gas ratios (DGR) are listed in Table 1. Equivalent radial distances from the galactic center were measured by assuming the position angle and axial ratio defined by Muñoz-Mateos et al. (2009). In the left panel of Fig. 7 the obtained DGR values are plotted against the equivalent angular radius along the semi-major axis. The observed profile of M 81 calculated by Muñoz-Mateos et al. (2009) is overplotted for comparison. It is evident that at distances $r^{\prime}>200^{\prime \prime}$ the DGR shows the typical decreasing trend with galactocentric distance. Note that the DGR values measured along Arp's loop are significantly higher than those measured in the central region of M81 and deviate from the general radial trend. It is interesting to note that the maximum DGR $\left(\log \left(M_{\text {dust }} / M_{\text {gas }}\right) \sim 0.12\right)$ is found in the northern side of Arp's loop, where redder MIPS colors have been measured and the HI emission drops below the detection limit. Such a large ratio has never been observed in any galaxy analyzed by Muñoz-Mateos et al. (2009) regardless of the distance from the galactic center. On the other hand the regions along Arp's loop with smaller DGR values are those located in the north-eastern part of the loop (where UV complexes have been observed; De Mello et al. 2008) and in the north-western part (where the spiral arm of M 81 overlaps Arp's loop). The surprisingly high DGR derived along Arp's loop results from the significantly small HI fluxes measured on the Walter et al. (2008) maps. To check the dependence of the obtained results on the adopted dataset, we calso alculated the DGR using the HI map provided by Yun et al. (1994). As apparent in Fig. 7, the derived DGR are very similar to those obtained using the Walter et al.'s map. Smaller intensities of the $21 \mathrm{~cm}$ emission in the eastern and northern part of Arp's loop are also evident in the single-dish map obtained by Appleton \& van der Hulst (1988). Moreover, the HI map of Walter et al. (2008) has been constructed using VLA data that include C and $\mathrm{D}$ configurations. On the basis of all these considerations, we can exclude the possibility that the high measured DGR could be assigned to sensitivity problems and/or filtering by the interferometer in the Walter et al. (2008) data. The high DGR measured along Arp's loop supports the idea that the far-infrared emission is increased due to the contribution of a dust-dominated source. In this regard, the hypothesis about Galactic cirrus fits this picture because it would provide a significant contribution to the far-infrared light, but its $\mathrm{HI}$ emission would be filtered out in the VLA maps of THINGS.

\section{Discussion}

We present a multi-wavelength analysis of Arp's loop using the deepest optical and infrared images available.

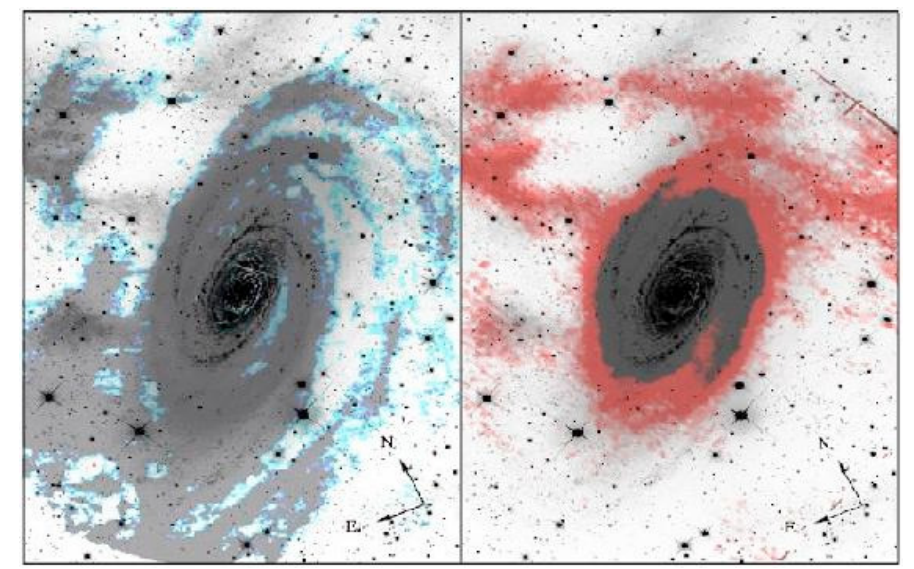

Fig. 5. HOA images of the region around M 81. The HI emission from Walter et al. (2008) (left panel) and the MIPS $160 \mu \mathrm{m}$ image (right panel) have been overplotted.

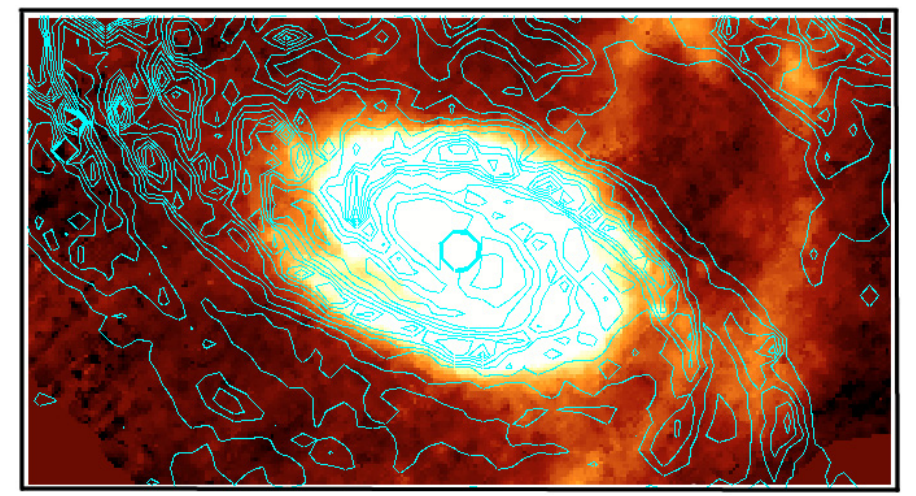

Fig. 6. MIPS $160 \mu \mathrm{m}$ image of M 81 . The contours of the HI emission from Walter et al. (2008) have been overplotted.

Arp's loop appears to be formed by a filamentary wrap that partially overlaps the disk of M 81. The many dust-absorption features overlapping M 81's field-of-view seen in the HOA images suggest that part of it is situated between the observer and the galaxy.

The comparison between the ratio of far-infrared fluxes emitted at $70 \mu \mathrm{m}$ and $160 \mu \mathrm{m}$ by Arp's loop and M 81 indicates that the emission from the loop is dominated by the contribution of cold dust. Its far-infrared emission resembles the properties of Galactic cirrus clouds, which also share the same colors in the IRAS bands. The same conclusion is also supported by the analysis of the DGR measured along Arp loop's extension, which indicates a surprisingly large relative fraction of dust if compared with that expected at such a large distance from the galactic center. It is therefore likely that at least part of the ring-like structure that forms Arp's loop is constituted by Galactic cirrus overlaying the disk of $M 81$. The region around M 81 is indeed known to be an area of great confusion between Galactic and extra-Galactic objects. Our observations cannot exclude stripped material due to the interaction with M 82 and NGC 3077 even in the regions of Arp's loop with small $I_{70 \mu \mathrm{m}} / I_{160 \mu \mathrm{m}}$ and high DGR. However, this process should have preferentially stripped dust, which is unlikely based on the predictions of the simulations of galaxy interactions (Yun 1999). Also in this scenario a contamination from the surrounding Galactic cirrus would help to explain all the observational evidences if it were overlapping Arp's loop. 
Table 1. Far-infrared fluxes and dust-to-gas ratios

\begin{tabular}{|c|c|c|c|c|c|c|c|c|c|c|}
\hline Location & $\begin{array}{c}\text { RA } \\
\text { h m s }\end{array}$ & $\begin{array}{c}\text { Dec } \\
\text { deg } \mathrm{m} \mathrm{s}\end{array}$ & Diameter & $\begin{array}{c}F_{60 \mu \mathrm{m}}^{\mathrm{IRIS}} \\
\mathrm{Jy}\end{array}$ & $\begin{array}{l}F_{100 \mu \mathrm{m}}^{\mathrm{IRIS}} \\
\mathrm{Jy}\end{array}$ & $\begin{array}{l}F_{70 \mu \mathrm{m}}^{\mathrm{MIPS}} \\
\mathrm{Jy}\end{array}$ & $\begin{array}{l}F_{160 \mu \mathrm{m}}^{\mathrm{MIPS}} \\
\mathrm{Jy}\end{array}$ & $\begin{array}{l}M_{\text {dust }} \\
10^{7} M_{\odot}\end{array}$ & $\begin{array}{l}M_{\mathrm{gas}} \\
10^{7} M_{\odot}\end{array}$ & $\log \left(M_{\text {dust }} / M_{\text {gas }}\right)$ \\
\hline Arp's loop NW & 095434 & +691654 & 4 & 0.962 & 3.493 & 0.285 & 4.386 & 0.393 & 9.043 & -1.362 \\
\hline Arp's loop N & 095543 & +691928 & 4 & 0.953 & 2.953 & 0.037 & 2.171 & 2.075 & 1.586 & 0.117 \\
\hline Arp's loop NE & 095702 & +691856 & 4 & 0.903 & 2.877 & 0.106 & 3.670 & 1.390 & 14.207 & -1.009 \\
\hline Arp's loop E & 095709 & +691121 & 4 & 0.853 & 2.788 & 0.198 & 3.048 & 0.271 & 2.6476 & -0.990 \\
\hline M 81's disk & 095533 & +690356 & 12 & 20.101 & 65.550 & 71.98 & 272.300 & 2.520 & 175.790 & -2.638 \\
\hline
\end{tabular}

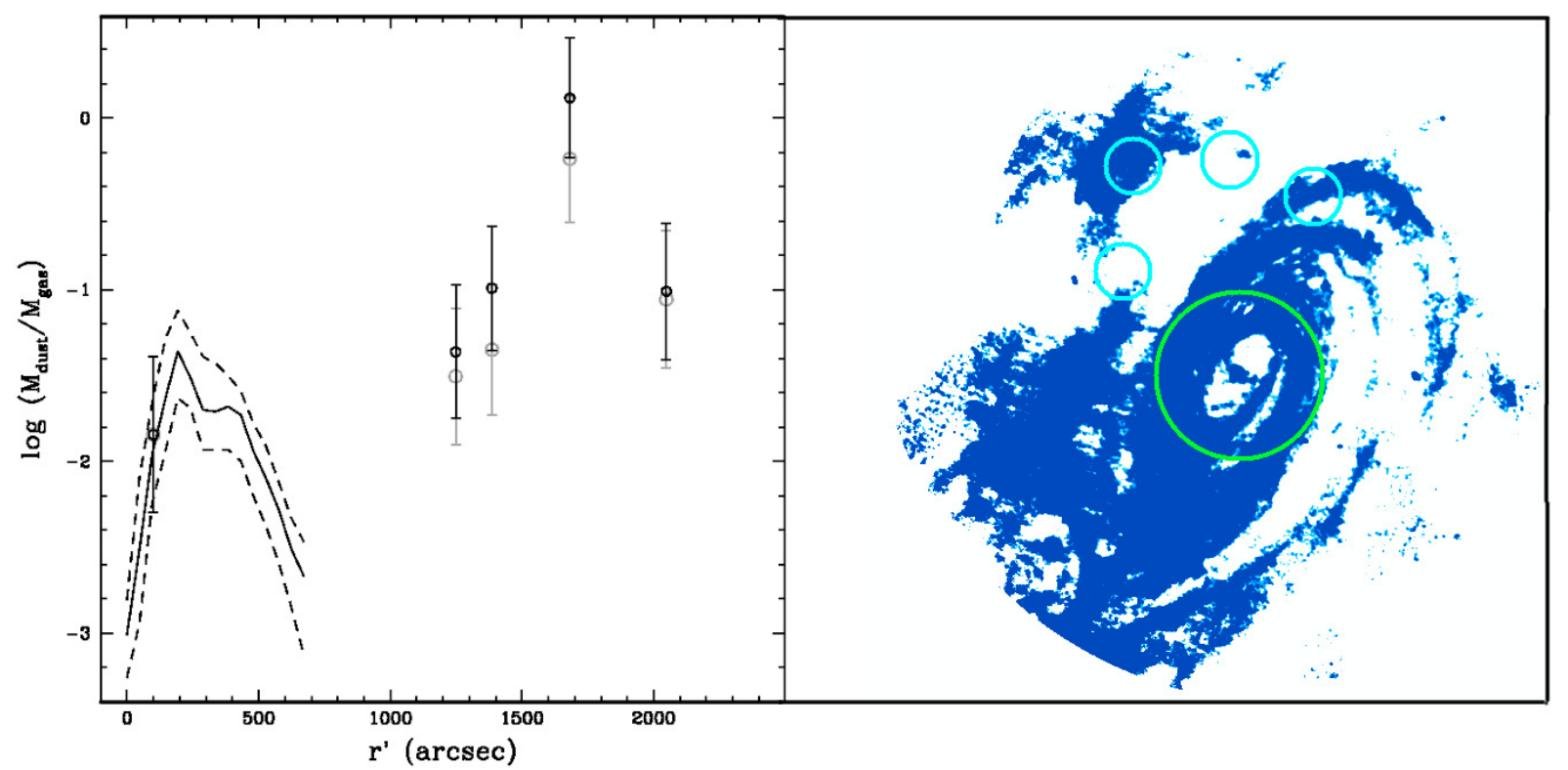

Fig. 7. In the left panel the DGR calculated for Arp's loop and the M 81 disk are shown as a function of the equivalent angular distance from the galactic center. Neutral hydrogen masses were calculated using both the Walter et al. (2008; black points) and Yun et al. (1994; grey points) HI maps. The radial DGR profile measured by Muñoz-Mateos et al. (2009) is overplotted. In the right panel the THINGS image of M 81 is shown. The circular apertures are also shown with the same color code as in Fig. 3.

On the other hand, an unambiguous distinction between these two possibilities is very complex (see e.g. Cortese et al. 2010) and cannot be made solely on the basis of morphological arguments or in terms of optical colors. In fact, cirrus clouds and galaxies have largely overlapping colors (Bremnes et al. 1998) and emissions that encompass a wide range of wavelengths from the UV to the far-infrared (Haikala et al. 1995).

In this context it is important to distinguish between the stellar populations surrounding M 81 and the nebular region which constitutes Arp's loop. In recent years, many sites of recent star formation have been reported in M 81's outer disk by GALEX (de Mello et al. 2008). Given the wide area covered by Arp's loop, it is possible that some of these star-forming regions could be accidentally located along the direct line of sight path to this feature. Regarding this, the spatial correlation between the HI emission and the Arp's loop provides some interesting evidence. As previously mentioned in Sect. 1, these HI clouds share similar dynamics with the disk of M 81. The deep photometric analyses of this region performed by Karachentsev et al. (2002), Makarova et al. (2002), Sun et al. (2005), De Mello et al. (2008), Davidge (2009) and Barker et al. (2009) revealed evidences for a young stellar population located at a distance comparable to M 81. Moreover, De Mello et al. (2008) identified eight FUV sources in this region using GALEX images. A stellar component associated with M 81 in this area therefore appears indisputable. However, notice that the HI emission disappears in the northern part of the loop where both optical and infrared images show significant contributions. Moreover, the analysis of CMDs obtained by Davidge (2009) and Barker et al. (2009) has not detected any overdensity of Red Giants along Arp's loop and only identified a population of young stars in the confined region where the strong HI emission was observed. Thus, although the origin of part of the optical and UV emission in Arp's loop could be emitted by recent star formation episodes, most likely part of it has a Galactic origin.

It is also remarkable that both $\mathrm{HI}$ and GALEX emissions extend across the entire disk of M 81 well beyond its optical cutoff, partially overlapping the north-western part of Arp's loop (see also Gil de Paz et al. 2007; Thilker et al. 2007). This was also confirmed by the analysis of HST CMDs which revealed the M 81's stellar disk population where GALEX and far-infrared emissions overlap (Gogarten et al. 2009).

The observational evidence put forward in this paper suggests that the structure known as Arp's loop is likely formed by three distinct components: i) one is associated with the M 81 system that causes the emission detected in the UV and HI plus part of the emission seen at optical wavelengths, thus dominating the morphology of the north-eastern part of the loop; ii) a second is dominated by M 81's extended disk, which contributes to the UV and HI emission in the north-western section of Arp's loop and; iii) another of Galactic origin that dominates the far-infrared emission and is responsible for the optical morphology (through scattering by dust) of the entire ring-like structure. 
Acknowledgements. This research was supported by the Spanish Ministry of Science and Innovation (MICINN) under the grant AYA2007-65090. We thank the anonymous referee for his/her useful comments and suggestions. We are grateful to Ignacio Trujillo, Michele Bellazzini, Julianne Dalcanton, and Juan Carlos Muñoz-Mateos for helpful discussions. We also thank Steve Mandel for providing us their wide field deep images of the M 81 sky area.

\section{References}

Abergel, A., Boulanger, F., Mizuno, A., \& Fukui, Y. 1994, ApJ, 423, L59 Abolins, J. A., \& Rice, W. L. 1987, NASA Conference Publication, 2466, 107 Appleton, P. N., \& van der Hulst, J. M. 1988, MNRAS, 234, 957

Appleton, P. N., Siqueira, P. R., \& Basart, J. P. 1993, AJ, 106, 1664

Arendt, R. G., Odegard, N., Weiland, J. L., et al. 1998, ApJ, 508, 74

Arp, H. 1965, Science, 148, 363

Barker, M.., Ferguson, A. M. Ñ., Irwin, M., Arimoto, N., \& Jablonka, P. 2009, AJ, 138, 1469

Boissier, S., Boselli, A., Buat, V., Donas, J., \& Milliard, B. 2004, A\&A, 424, 465

Bot, C., Helou, G., Boulanger, F., et al. 2009, ApJ, 695, 469

Bremnes, T., Binggeli, B., \& Prugniel, P. 1998, A\&AS, 129, 313

Carey, S. J., Shipman, R. F., Clark, F. O., \& Assendorp, R. 1997, ApJ, 479, 303

Cortese, L., Bendo, G. J., Isaak, K. G., Davies, J. I., \& Kent, B. R. 2010, MNRAS, 403, L26

Dale, D. A., Gil de Paz, A., Gordon, K. D., et al. 2007, ApJ, 655, 863

Davidge, T. J. 2009, ApJ, 697, 1439

Davies, E. 1990, in Machine Vision: Theory, Algorithms and Praticalities, (Academic Press), 42 de Mello, D. F., Smith, L. J., Sabbi, E., et al. 2008, AJ, 135, 548

Efremov, Y. N., Karachentsev, I. D., \& Karachentseva, V. E. 1986, Pis ma Astronomicheskii Zhurnal, 12, 434

Gil de Paz, A., Boissier, S., Madore, B. F., et al. 2007, ApJS, 173, 185

Gogarten, S. M., Dalcanton, J. J., Williams, B. F., et al. 2009, ApJ, 691, 115

Gordon, K. D., Rieke, G. H., Engelbracht, C. W., et al. 2005, PASP, 117, 503

Gordon, K. D., Engelbracht, C. W., Rieke, G. H., et al. 2008, ApJ, 682, 336

Gottesman, S. T., \& Weliachew, L. 1975, ApJ, 195, 23

Haikala, L. K., Mattila, K., Bowyer, S., et al. 1995, ApJ, 443, L33

Helou, G. 1986, ApJ, 311, L33

Issa, M. R., MacLaren, I., \& Wolfendale, A. W. 1990, A\&A, 236, 237

Karachentsev, I. D., Karachentseva, V. E., \& Huchtmeier, W. K. 2001, A\&A, 366,428

Karachentsev, I. D., Dolphin, A. E., Geisler, D., et al. 2002, A\&A, 383, 125

Kennicutt, R. C., Jr., Armus, L., Bendo, G., et al. 2003, PASP, 115, 928

Lagache, G., Haffner, L. M., Reynolds, R. J., \& Tufte, S. L. 2000, A\&A, 354, 247

Makarova, L. N., Grebel, E. K., Karachentsev, I. D., et al. 2002, A\&A, 396, 473

Mouhcine, M., \& Ibata, R. 2010, MNRAS [arXiv: 1002 .0456]

Muñoz-Mateos, J. C., Gil de Paz, A., Boissier, S., et al. 2009, ApJ, 701, 1965

Sandage, A. 1976, AJ, 81, 954

Schlegel, D. J., Finkbeiner, D. P., \& Davis, M. 1998, ApJ, 500, 525

Sun, W.-H., Zhou, X., Chen, W.-P., et al. 2005, ApJ, 630, L133

Thilker, D. A., Bianchi, L., Meurer, G., et al. 2007, ApJS, 173, 538

Walter, F., Brinks, E., de Blok, W. J. G., et al. 2008, AJ, 136, 2563

Witt, A. N., Mandel, S., Sell, P. H., Dixon, T., \& Vijh, U. P. 2008, ApJ, 679, 497

Yun, M. S. 1999, in Galaxy Interactions at Low and High Redshift, ed. J. E.

Barnes, \& D. B. Sanders, Proc. IAU Symp., 186, 81

Yun, M. S., Ho, P. T. P., \& Lo, K. Y. 1994, Nature, 372, 530 